\title{
GLOBAL CHALLENGES IN THE REGULATION OF AQUACULTURE
}

\author{
William Howarth \\ Professor of Environmental Law, \\ University of Kent at Canterbury \\ Eliot College, University, Canterbury, Kent, CT2 7NS, United Kingdom \\ W.Howarth@ukc.ac.uk
}

\section{Objectives}

The purpose of this paper is to sketch out some broad themes concerning the regulation of aquaculture. The view taken is that aquaculture is an activity which has enormous potential to enhance food resources and laws are needed to facilitate the realisation of this potential with the least possible impediment. Nonetheless, there are a range of environmental and social concerns that need to be reconciled with aquaculture development. The legal challenge is that of securing the optimum balance of interests consistent with the imperative of sustainable development and using the range of legal approaches in the manner which best reflects the gravity of the concerns which need to be safeguarded against. Broadly, the paper progresses from some general observations about the relationship between aquaculture and law into a discussion of some of the more specific issues that need to be addressed. In the latter part of the discussion, a number of illustrations are introduced of legal mechanisms that have been employed in the United Kingdom, and particularly in Scotland. These examples are intended to illustrate regulatory possibilities, and are not presented as definitive legal solutions to the issues under discussion.

\section{Introduction}

Perhaps the greatest and most enduring challenge for humanity is that of feeding itself. Since the dawn of time, human beings have struggled to find a balance between the extremes of surplus and shortage found in nature. That struggle continues today, with an alarmingly large proportion of the world still suffering food shortages.

Insofar as security of food supplies is the greatest challenge for humanity, agriculture might be seen as its greatest achievement. On the time-scale of humanity, we have progressed relatively recently from hunters and gatherers of our food, dependent entirely upon the bounties and vagaries of nature, to societies that know how to feed themselves by growing the crops and keeping the livestock that provide the bulk of our food supplies. Security of food supplies has provided the bedrock upon which all subsequent technological and other development has been made possible.

The triumph of agriculture, however, deserves a moment's reflection. How did this come about? Certainly, by thousands of years of trial and error by individuals and communities which long predated modern 'scientific' knowledge about the most productive methods of cultivating plants and rearing animals. The techniques of 
agriculture are a ancient heritage of hard learnt lessons from persons who faced starvation because of their mistakes and cherished their successes for good reason.

Equally, agriculture is a lesson in the way that human beings must organise their activities for mutual benefit. There could have been no incentive for people to plant crops, without the expectation that after months of painstaking work tending them, they would be assured the end product at the time of harvesting. Agriculture is only possible on the basis of a system of land tenure which allows its participants a sufficient degree of ownership over the products of their labour. Where there is a surplus of suitable land this may not be problematic, but in almost all societies of which we have knowledge, the need for defined land rights is a precursor to any almost any kind of productive agricultural activity. The achievements of agriculture rest upon the ability to define and protect property interests as much as they have depended upon the development of practical farming techniques. Without the former, the latter would have been futile.

\section{Food From Waters}

Turning from the production of food from land to the production of food from waters, the primitiveness of our present situation is apparent. Technological advancement has preceded, rather than followed, organisational development. Sufficient fishery know-how has been acquired to denude the world's oceans of almost every fish that it is commercially worth catching. What has only recently come to be generally appreciated is that the first-come-first-served approach to fishery resources may not be genuinely conducive to the long term interests of humanity. Moreover, to the extent that it is not conducive to those interest, humanity is only in the relatively early stages of establishing a system of rights that is appropriate for protecting the continuing productivity of the sea against the ravages of excessive exploitation. In short, common resources provided by nature need to be subjected to legal regimes which allocate rights of exploitation to ensure that sustainable yields are not exceeded and that those yields are equitably shared for the general benefit.

The warning was issued in 1968 with the publication of Garrett Hardin's famous essay on 'The Tragedy of the Commons' in which he envisaged that 'the inherent logic of the commons remorselessly generates tragedy' by maximising individual gain to the collective detriment so that 'freedom in a commons brings all to ruin'. The validity of this thesis is hotly disputed, but in the case of fisheries common property in natural resources has enabled a ruinous decline in global fisheries to take place. It would be good to think that humanity has the good sense to address this by mutual self regulation, but many would take the view that the response has been too little too late.

Although, it is encouraging to see recent international co-operation on fisheries, and particularly the need to address conservation of migratory stocks of fish which move

The author is pleased to acknowledge help with the preparation and revision of this paper from Annick Van Houtte of the Development Legal Service of the Food and Agriculture Organization of the United Nations and Professor David VandersZwaag of Dalhousie Law School.

1 G. Hardin, 'The Tragedy of the Commons' (1968) 162 Science 1243. 
between waters within the jurisdiction of different states, ${ }^{2}$ much more needs to be done. International fisheries regulation is upon the cusp of moving from pure hunter-gathering into a new era of managed international natural resource regulation. The fact remains that most terrestrial agriculture passed through this point of development several thousand years ago.

\section{The Birth of Aquaculture}

Where does aquaculture feature on this scale of development? Certainly, it has undergone massive scientific and technological advancement in an astonishingly short space of time. Although awareness that containment of fish within ponds was a means of securing supplies in times of shortage can be traced back to ancient times, ${ }^{3}$ the capacity to breed and culture fish, and significantly to enhance natural productivity, is a modern discovery. Within a small number of decades, developed countries have surmounted the problems of rearing fish in marine waters and have transformed salmon from a luxury product to a relatively cheap source of food, at least in developed countries. The cultivation of shellfish has developed unrecognisably in the space of a few years and, again, products have become available with a security that would not have been previously imagined. The prospects for the future look to be even more encouraging, with the increasing sophistication of culture techniques and installations, and the prospect of farming an increasing range of species.

The remarkable success story evidenced by the recent development of aquaculture provides convincing evidence that this is route for future development. The shortcomings of capture fisheries and the progressive decline in catches, despite increased fishing effort, is capable of being perceived as the last throws of an industry which is in the process of being gradually superseded. The vision for the future is of seas which will be 'farmed', with progressive reductions in reliance upon purely natural fish stocks. It must be accepted that this prospect is likely to be rather too futuristic for some, at the present at least, but if the analogy with agriculture is a fair one it may not be entirely fanciful.

The recent achievements in aquaculture have been nothing short of miraculous. Compared to the thousands of years which farming took to evolve to its present state, the farming of fish and shellfish has developed 'overnight'. However, whist the achievement should not be diminished, neither should it be overlooked that it has come at a cost. The dazzling technical achievement of aquaculture has been followed by a more reflective period of assessing the impacts and costs, and perhaps a sceptical feeling in the minds of some spectators that the achievement is simply too good to be true.

The sceptical observer does not need to look too hard for a down side. Aquaculture inevitably involves an intrusion into the natural environment which leads into a

\footnotetext{
${ }^{2}$ Generally see Food and Agriculture Organization of the United Nations Legal Office, Law and Sustainable Development Since Rio (Rome: FAO, 2002) particularly Ch.7 on fisheries.

${ }^{3}$ Generally see R. Kirk, A History of Marine Fish Culture in Europe and North America (Farnham:

Fishing News Books, 1987).
} 
catalogue of environmental quality and ecological adversities. Any perception that aquaculture involves 'something for nothing', is swiftly dismissed. The evidence substantiating a range of actual environmental, economic and social adversities is considerable, and the evidence for an range of other potential harms is at least plausible, if presently lacking in substantiation by research. The second wave issues are about how the balance of benefits and costs is most appropriately drawn.

\section{The Role of Regulation}

Lawyers always have to tread carefully when wading into this kind of exercise. Many of the issues are most centrally placed within the remit of environmental scientists, who must make expert determinations as the extent and gravity of environmental impacts. Insofar as the benefits and impacts of aquaculture development can be properly assessed, they provide boundaries which need to be reflected in regulation. The task of the lawyer is that of trying to reflect the balance of costs and benefits in regulation which facilitates the development of the industry whilst providing the safeguards which are needed against unacceptable environmental, and other kinds of, intrusion. The knee jerk reaction to any kind of adversity, that 'there ought to be a law against it', should be avoided. Even where regulation provides an appropriate response to a problem, it must be appreciated that laws are capable of being formulated in an infinite variety of ways. The test of a good law is how well it reflects the balance of interests involved in defining the boundary between the permissible and the impermissible.

Clearly, there is conviction that the free-for-all in respect of capture fishery resources, which has prevailed until fairly recent times, should not serve as a model for a free-for-all in respect of aquaculture development. The present focus is upon regulation which facilitates the new industry, to allow it to provide the substantial benefits to humanity which are within its potential, but ensures that its environmental and social impacts are brought within acceptable bounds.

\section{Sustainable Development}

Inevitably, since the Rio Conference on Environment and Development in 1992, it is necessary to look to the regulatory touchstone provided by the imperative of 'sustainable development': defined in the Brundtland Report of the World Commission on Environment and Development as "development which meets the needs of the present without compromising the ability of future generations to meet their own needs". 4

The critics and sceptics of sustainable development have drawn attention to the open-ended and obscure nature of the international imperative, and their observations are difficult to refute. Nonetheless, in the absence of anything better, we are stuck with the concept sustainable development as a guide to the way in which we should regulate all kinds of developmental activity to ensure that they take place at an acceptable environmental cost. What is useful about the concept is that

\footnotetext{
${ }^{4}$ World Commission on Environment and Development, Our Common Future (Oxford: Oxford University Press, 1987) p.43.
} 
it recognises that development has a cost, as well as a benefit, and that this cost should be minimised and not allowed to exceed the value of environmental goods that are lost as a result of development.

\section{Elaboration of Sustainable Development}

The concept of sustainable development, however, should always be seen as a starting point rather than a point of destination. The concept needs to be reinterpreted by each nation in the specific context of its developmental needs. Within nations, the reinterpretation needs to be taken a stage further in identifying what the concept means for each sector of activity, including fishery and aquaculture development.

Some important international work has been done in reinterpreting sustainable development in relation to aquaculture activities. The Food and Agriculture Organization's Code of Conduct for Responsible Fisheries ${ }^{5}$ is a valuable, though largely voluntary, global statement of principles addressed to governments, organisations and individuals engaged in fishery activities which seeks to encourage adherence to principles and standards conducive to sustainability in the conservation, management and development of fisheries.

Article Nine of the FAO Code, concerned specifically with aquaculture development, encourages states to promote sustainability in aquaculture by the use of strategic planning to take account of, and minimise, adverse environmental and social impacts and to adopt a co-operative approach towards transboundary impacts. At a national level, this involves conservation of genetic diversity and integrity of ecosystems by encouragement of appropriate practices and procedures. At the operational level, promotion of responsible aquaculture should involve encouragement, and in some respects, the regulation of chemical inputs which are hazardous to human health and damaging to the state of the environment. Not least important, is the need to prevent aquaculture practices which are actually harmful to aquaculture industry itself, such as the need to prevent disease transmission and to ensure food safety in aquaculture products. The potentially catastrophic implications of failure to ensure protection against these matters will be self-evident to all who are involved.

Further elaboration of the practical implications of the general principles set out in the Code of Conduct on Responsible Fisheries are provided in Food and Agriculture Organization's Technical Guidance for Responsible Fisheries. ${ }^{6}$ In addition, further international guidance on sustainability in aquaculture is provided by the Holmenkollen Guidelines for Sustainable Aquaculture, ${ }^{7}$ which are addressed to governmental authorities and those actively involved in aquaculture, and identify key issues needing to be addressed in securing greater sustainability. Also, the

\footnotetext{
${ }^{5}$ Food and Agriculture Organization of the United Nations, Code of Conduct for Responsible Fisheries (Rome: FAO, 1995).

${ }^{6}$ Food and Agriculture Organization of the United Nations, FAO Technical Guidelines for Responsible Fisheries: 5 Aquaculture Development (Rome: FAO, 1997).

7 See N. Svennevig, H. Reinersten and M. New, Eds, Sutainable Aquaculture: Food for the Future? (Rotterdam: A. A. Balkema, 1997) particularly p.343 onwards.
} 
Bangkok Declaration and Strategy for Aquaculture Development beyond $2000^{8}$ offers insightful observations as to key policies which states should pursue to enhance sustainability in aquaculture.

However, it is in the nature of international guidelines that they must be couched as generally formulated approaches to the environmental, ecological and social problems which may be misread as indicating all-purpose solutions for a diversity of national and local situations and activities. This diversity in practice is clearly a practical strength of aquaculture, in providing widely different responses to meet different local needs. Hence, internationally established principles and proposals must always have their limitations insofar as they are drafted remotely from the actual circumstances and activities which need to be influenced or regulated. The international guidance always needs to be reinterpreted in the light of local conditions and in the context of the particular activities that are being undertaken.

\section{Developmental Relativity}

Arguably, the valuable work undertaken by international organisations might be seen as posing, rather than answering, the pertinent questions which individual states must address in organising their relevant activities. The point needs to be stressed that no two nations are at an identical state of development and the environmental balance sheet needs to be the subject of national introspection. As a generality, sustainable development implies that more developed countries should place a greater weight upon environmental goods than less developed countries, but how this balance sheet should be drawn up is a matter for each state to determine.

I was fortunate to be engaged to work with the legal staff of the Food and Agriculture Organisation a couple of years ago on a project looking at the regulation of shrimp farming in developing countries. ${ }^{9}$ The team of us who were involved in the project sought information about the state of regulation of aquaculture in some twenty different countries in Asia, East Africa and South America to identify some pointers towards best practice in the sector and to offer some suggestions as to regulatory and administrative measures which might be seen as desirable in developing countries.

The shrimp legislation project was a revealing one, in showing the spectrum of approaches adopted by a range of countries which, within limits, might be seen as being at comparable states of development. Each of them had a pressing need for the economic benefits provided by shrimp farming, but also had legislation reflecting varying levels of concern as to the extent which economic benefits should be allowed to damage their natural environments. It was encouraging to see that some states had taken account of the need for sustainable development in formulating

\footnotetext{
${ }^{8}$ Network of Aquaculture Centres in Asia-Pacific and Food and Agriculture Organization of the United Nations, Aquaculture Development Beyond 2000: The Bangkok Declaration and Strategy (Rome: NACA/FAO, 2000).

W. Howarth, R. Hernandez and A. Van Houtte, Legislation Governing Shrimp Aquaculture - Legal Issues, National Experience and Options (Rome: FAO, 2001) and see http://www.fao.org/Legal/prsol/paper-e.htm.
} 
policies and legislative measures which sought to facilitate shrimp farming development, but had allowed this in a way which minimised environmental and ecological harm. Even where seriously limited resources were available to particular states, it was seen to be appropriate to devote these resources to mitigating environmental impacts.

Turning to developed countries, like Canada or the United Kingdom, environmental expectations should be all the higher. Generally, sustainable development envisages that strong economies are expected to take the lead on matters of environmental performance. Adverse environmental and ecological impacts, that might be justified by extreme developmental needs in developing countries, should not be acceptable in developed countries where the threat of poverty and starvation cannot be provided as a justification for environmental damage. Hence, developed countries should be aiming for the highest environmental standards.

\section{The Environmental Checklist}

The 'checklist' of possible environment impacts, which may arise from aquaculture and need to be addressed, is not too difficult to ascertain from examining the issues covered by the international initiatives. Key general issues include: environmentally or ecologically destructive land use; unacceptable impacts on water resources either through abstraction or the release of contaminated discharges; damaging ecological impacts upon natural resources through the excessive removal of specimens from the wild or the release of farmed species into the wild; the transmission of disease to farmed stocks and natural ecosystems by the unrestrained movement of farmed stocks; and a range of intrusions upon water and land uses which conflict with legitimate interests of others. ${ }^{10}$

Again, the international literature needs to be read with caution. Although useful as a checklist of the problems that may need to be addressed, it needs to be equally appreciated that aquaculture activity is remarkably diverse. Small scale, low intensity, fish farming operations in developing countries are capable of having almost negligible environmental or ecological impacts, and need to be regulated accordingly. Where economic circumstances preclude the use of pesticides and chemicals in aquaculture, or the importation of non-native species, it may not be necessary to devise elaborate systems of regulatory control to address a problem that does not exist in practice.

However, the intensive and large scale approach to aquaculture taken in developed countries is likely to place it in a different regulatory category. The onus is upon each state to ascertain to what extent the potential environmental problems associated with aquaculture are actually evidenced as consequences of the kinds of operation that take place within its jurisdiction. Large scale, sea cage installations, perhaps farming non-native species and subjecting stocks to sophisticated programmes of medication, give rise to a wide range of potential environmental and ecological risks. Clearly, the extent to which risks are capable of being converted

${ }^{10}$ Generally see T. V. R. Pillay, Aquaculture and the Environment (Oxford: Fishing News Books, 1992). 
into harms requires extensive investigation and research to be undertaken. Where research of this kind has not been undertaken, legislation may be justified on preventative or precautionary grounds.

\section{The Regulatory Response}

The central issue is the role of the law in seeking to provide for a balance between aquaculture development and environmental protection. On this, a personal conviction has to be expressed that the best kind of regulation is self-regulation. A belief that something should be done because it is right to do it, is always more effective than a mandatory legal prohibition backed by a sanction. In the field of aquaculture, the paths of self-regulation and self-interest follow close parallels. It is not in the interests of fish farmers to contaminate the waters upon which their livelihood depends, to allow their stock to become tainted by pesticides or other chemicals, or to alienate their consumers by engaging in anything less than environmentally exemplary performance. In all the main respects, commercial good sense and environmental good sense run along the same lines.

It is evident that the interests of the aquaculture industry as a whole, may not be the same as the interests of particular fish farmers who must compete for commercial advantage against their fellow producers. The incentives to secure greater profitability is capable of motivating individual fish farmers to 'cut corners' at the expense of the environment, but the obvious first-resort mechanism to address this is through measures imposed by the industry upon its members.

The role of producer groups in securing cohesion within the aquaculture industry is fundamental. Uniformly high standards may be facilitated through self-education, product and practice standards, perhaps by the establishment of codes of practice, which determine what activities should be adhered to by all participants. ${ }^{11}$ The incentives for adherence to those standards by particular fish farmers is that their product will be seen to have the certifiably high quality stipulated by the industry as a whole. The counterpart of this is that producer groups should have the power to exclude from their membership any producer who is not willing to adhere to the standards set by the industry for itself. Given recent experiences of 'food scares' in diverse sectors, the desirability of any food producers group taking measures to safeguard the quality of its products seems justifiable. It only needs one fish farmer to misuse chemicals in treating stock to blight the industry as a whole, and the power to dissociate the non-compliant farmer from the quality assurance standards promulgated by the industry as a whole seems justifiable.

A good illustration of the benefits that may be secured by cohesion within the aquaculture industry is to be seen in the 'Scottish Quality Salmon' quality assurance scheme. This scheme requires members to be certified as complying with both product standards and environmental management schemes that are independently

\footnotetext{
11 Generally see C. Hough, 'Codes of Practice and Aquaculture' in R. Leroy Creswell and R. Flos, Eds., Perspectives on Responsible Aquaculture for the New Millennium (Louisiana and Oostende: World Aquaculture Society and European Aquaculture Society, 2002) p.79.
} 
accredited to international standards, such as ISO $14001 .^{12}$ Hence, the benefits to producers of a recognised status being attached to a product, entail a commitment to adhere to high environmental standards throughout the production process. In addition, the Scottish aquaculture industry is in the process of developing a Code of Best Practice (covering, disease control, welfare, and health and safety) and within that a Code of Best Environmental Practice. The intention is that every licensed aquaculture operator will be expected to be formally subscribed to these codes by 2004 and compliance will be externally monitored and audited. ${ }^{13}$

Alongside action taken at an industry-wide level, significant benefits can be achieved by local cooperation between individual fish farmers. An example of such co-operation in Scotland is the increasing co-ordination of restocking, medication and fallowing operations. It is recognised that breaking potential disease cycles, by minimising the risk of cross infection, and preventing 'souring' of the seabed beneath cages requires areas to be left fallow for periods. However, for this to be effective, close liaison between neighbouring fish farms is needed. The indications are that voluntary management agreements have been successfully reached between neighbouring fish farmers to reduce the possibilities of adverse interaction. These inter-company agreements have produced mutual benefits to operators and have reduced environmental impacts. ${ }^{14}$

Education and the formulation of standards or codes of practice should involve close collaboration between producers groups and government. A code of practice promulgated by government has an additional status in that it can be used as a prerequisite for other kinds of action. For example, an undertaking that a prospective fish farmer will adhere to the terms of the code might be required as a condition in a licence for a particular operation or as a prerequisite for that some kind of grant or fiscal advantage being made available to a particular fish farmer. Hence, it is possible to interlink a code with other kinds of benefit to enhance its importance.

Self-regulation by producers groups or in co-operation with government, though important, is not without its limitations. The issue remains as to what legal response is needed at the point where self-regulation runs out, or perhaps where legislation is seen as an important means of bolstering support by self-regulation. When it comes to regulation, properly so called, it is not a simple matter of prohibiting and providing sanctions for each activity which is perceived to be problematic and not adequately

12 Scottish Executive, Environment and Rural Development Department, A Strategic Framework for Scottish Aquaculture (Draft), (Edinburgh Scottish Executive, 2002) para.3.83 (see

http://scotland.gov.uk). Generally see European Aquaculture Society, Certification, Labelling and Quality of European Aquaculture Produce, Publication 163 (Brussels:EAS, 2002) and L. M. Laird, 'Quality Approaches as Guarantees for responsible Aquaculture, in R. Leroy Creswell and R. Flos, Eds., Perspectives on Responsible Aquaculture for the New Millennium (Louisiana and Oostende: World Aquaculture Society and European Aquaculture Society, 2002) p.49.

${ }^{13}$ Scottish Executive, Environment and Rural Development Department, $A$ Strategic Framework for Scottish Aquaculture (Draft), (Edinburgh Scottish Executive, 2002) para.3.83 (see http://scotland.gov.uk) para.3.93.

${ }_{14}$ Scottish Executive, Environment and Rural Development Department, Advice Note: Marine Fish Farming and the Environment, (Edinburgh Scottish Executive, 2003) (see http://scotland.gov.uk) para. 67 onwards. 
addressable by self-regulation. In each instance, the task is that of identifying a legal response which is appropriate and proportionate as the most likely mechanism to secure the desired policy objective. The diversity of possible regulatory approaches deserves careful consideration.

\section{Regulatory Diversity}

From the outset, it must be appreciated that law is a remarkably flexible control mechanism. In each instance, a wide range of regulatory approaches are possible and the determination of which of these is appropriate requires a range of factors to be weighed into the balance. Legislation may be used comprehensively to prohibit an activity; to restrict the manner in which the activity is undertaken; to licence, permit or authorise the activity to enable it to be undertaken in limited circumstances (perhaps subject to locally-formulated conditions); to require the registration of an installation where an activity is conducted; or positively to compel (rather than prohibit) an activity to be undertaken. Moreover, legal foundations are frequently needed for information-based measures which facilitate research, monitoring, investigation, and educational measures which allow for the financial or technical support in relation to an activity.

This wide spectrum of potential legal responses needs to be stressed because of the misplaced tendency to suppose that the use of the law means, necessarily and exclusively, the banning and punishment of unacceptable practices. In fact it facilitates a diverse range of possible requirements, backed by a variety of sanctions and incentives, and important issues arise in weighing up the advantages and disadvantages of the different legal responses which are possible.

Recognising the potential flexibility of the legal response, the task for national authorities is that of relating the gravity of environmental problems generated by aquaculture to the appropriate level of legal coercion which is needed to address them. Broadly, it may be supposed that the most environmentally damaging environmental practices need to be made subject to the greatest degree of legal stringency, assuming that a legal response is found to be necessary to address the problem in the first place. At the other extreme a range of issues may be better addressed by non-mandatory mechanisms. Education and training are arguably as valuable as law in directing fish farmers towards good practice, and economic incentives which are made conditional upon good environmental performance may provide a greater incentive for avoiding environmental damage than the threat of legal proceedings and punishment.

\section{Initial Controls}

Categorised according to the point of impact, legal controls upon aquaculture may be subdivided into 'initial' and 'continuing forms of control'. Initial controls are concerned with the procedures which determine whether an aquaculture installation may be established at a particular location, whereas continuing controls are those measures which apply to subsequent activities which take place at the installation following its establishment. For good reasons, the questions, first, where a fish farm 
should be located and, second, how it should conduct its operations, are best approached sequentially. ${ }^{15}$

The issue of rights to land covered by water is of considerable legal complexity, particular where marine waters are at issue. Insofar as the seabed is not the subject of private ownership, it will be necessary to acquire a lease or similar authorisation from the public body with responsibility for sea bed operations. 'Development licensing' is capable of taking diverse legal forms, but essentially these all involve a system of public land use control which seeks to subsume particular lease determinations to broader strategic policies for development of areas or sectors of activity. ${ }^{16}$

The mechanisms for regulating developments of the seabed have recently been reconsidered in Scotland. Presently, it is necessary for a prospective fish farmer to obtain both a development consent and a licence from the Crown Estate Commissioners, who administer the Crown Estate including the seabed. ${ }^{17}$ For the future, it is envisaged that responsibility for development control will pass to local authorities, who will also have responsibility for environmental impact assessment where this is required. The involvement of the Crown Estate Commissioners will continue, so far as a lease for the site will be needed from the Commissioners. However, the move represents a shifting of control away from a body which, in the past, has been seen to be too closely associated with the commercial aspects of authorising a development, insofar as the Commissioners would be entitled to rent under any lease that was granted. Under interim arrangements, local planning authorities will advise the Commissioners on fish farm applications. ${ }^{18}$

It is for government, or perhaps regional or local licensing authorities, to determine strategic policies for land use, but this needs to be done as a result of open deliberation and widespread consultation about the issues to be taken into account. Aquaculture frequently has special requirements for suitable marine areas, perhaps because of the need to ensure sufficient water flows but to avoid excessive exposure under storm conditions. The difficulty is often that the same areas that are sought by prospective fish farmers are also most valued for traditional fishing, recreation, conservation or needed to be kept free of obstructions for the purposes of navigation. Insofar as it is possible to do so, an integrated development planning exercise will be needed for marine areas to identify those areas which are most suitable for the different kinds of activity and minimise the conflicts that are likely to arise between activities that compete for water space.

An illustration of a strategic approach to aquaculture planning is to be seen in the Scottish Executive Environment and Rural Affairs Department's recently revised

\footnotetext{
${ }^{15}$ Generally see B. Wildsmith, Aquaculture: The Legal Framework (Toronto: Edmond-Montgomery, 1982 ) and W. Howarth, The Law of Aquaculture (Oxford: Fishing News Books, 1990).

${ }^{16}$ Generally see T. V. R. Pillay, Planning of Aquaculture Development - an introductory guide (Farnham: Fishing News Books/FAO, 1977).

17 Under the Crown Estate Act 1961.

18 Scottish Executive, Environment and Rural Development Department, Advice Note: Marine Fish Farming and the Environment, (Edinburgh: Scottish Executive, 2003) (see http://scotland.gov.uk) paras.2 and 11.
} 


\section{Locational Guidelines for the Authorisation of Marine Fish Farms in Scottish}

Waters. $^{19}$ The purpose of these Guidelines is to provide guidance on the factors to be taken into account in considering proposals for fish farms and to encourage local authorities to prepare non-statutory marine fish farming framework plans, as a guide to the location of future fish farms. Drawing upon a range of recent ecological information, the Guidelines reaffirm a presumption against fish farm developments on the north and east coasts of Scotland. Elsewhere three categories of area are envisaged on the basis of nutrient loading and benthic impact: first, where fish farms will only be acceptable in 'exceptional circumstances', second, where (areas are at the limits of their carrying capacity) so that further development will result in these areas being placed in the first category, and, third, where there is a 'better prospect' of satisfying nutrient loading and bethic impact requirements. In summary, only those areas in the third category are likely to be acceptable for new fish farm development. Even then, for applications in category three areas, it is stated that the circumstances will need to be carefully considered before permission will be given, and proposals are only likely to succeed where they involve a package of measures designed to reduce environmental impact. Such measures, for example, will include feeding techniques which minimise food wastage and integrated sea lice management practices which reduce the use of chemical treatments.

Although strategic zoning approaches of this kind are helpful in guiding development towards areas that are seen to be least problematic, actual determinations of whether a particular project should be allowed in a particular zone need to be decided against a background of specific information about the nature of the project and its foreseeable impacts. Although much of this information falls to be provided by the developer, the determination process should seek to maximise involvement in the decision making process by encouraging representations to be made by relevant public bodies, organisations and members of the public. Where representations of this kind are made, it should be the responsibility of the decision making body to take these into account in the final decision.

For example, in Scotland, the consultation process surrounding an application for a development consent for a fish farm is an important mechanism for securing the opinion of the statutory nature conservation and landscape protection body, Scottish Natural Heritage. Scottish Natural Heritage is well placed to advise on a range of implications upon flora and fauna of fish farm applications. Notably in this respect, substantial areas of land are subject to conservation designation in accordance with national provisions or legislation adopted at European Community level, and it is important that aquaculture should be steered away from these areas. Another relevant aspect is the need to minimise adverse impacts with predator species of seabirds and mammals. In this respect, Scottish Natural Heritage is able to advise on avoidance of such conflicts, though it is also notable that the industry and

19 Scottish Executive, Environment and Rural Affairs Department, Locational Guidelines for the Authorisation of Marine Fish Farms in Scottish Waters (Edinburgh: Scottish Executive, 2003) (see http://scotland.gov.uk). 
conservation organisations have agreed a Code of Practice on the Interaction of Fish Farming and Predatory Wildlife. ${ }^{20}$

In particular determinations as to whether an aquaculture installation should be allowed at a particular location, environmental impact assessment has proved to be an invaluable tool in many jurisdictions. Essentially, the process involved requires the prospective developer to provide information about the intended project and to anticipate the range of impacts that it will have upon the surrounding environmental media and ecosystems and other activities that are conducted in the vicinity. The final determination is made on the basis of this information, and should seek to incorporate mechanisms to ensure that the adverse impacts are minimised to an extent which is acceptable, or the application is declined where sufficient mitigation is not possible. Hence, an eventual authorisation is likely to be hedged around with a list of conditions which seek to minimise adverse environmental consequences.

In the United Kingdom, regulation is provided for under Environmental Impact Assessment (Fish Farming in Marine Waters) Regulations $1999^{21}$ and associated guidance, which implement European Community requirements for environmental impact assessment. ${ }^{22}$ Regulations apply where any part of the development is within a 'sensitive area; where the development is designed to hold a biomass of 100 tonnes or greater; or where the proposed development will extend to 0.1 hectare or more. Nonetheless, there are concerns that environmental impact assessment is not functioning as well as it might in Scotland because of the relatively variable standard of environmental statements submitted in relation to fish farm applications and their failure to identify cumulative impacts of developments and discharges. ${ }^{23}$

Development licensing, in conjunction with environmental assessment, is, therefore, a very flexible legal tool, which may be applied with different degrees of rigor and sophistication depending upon national and local needs. In an extreme case, development licensing may allow the most comprehensive investigation of all environmental and social impacts to which a project may give rise. The licensing authority will have before it all the information that it needs to ascertain whether the project is consistent with the requirements of sustainable development. Accordingly, it may impose conditions of a preventative or precautionary kind where it feels that there are special risks that need to be secured against. Not least important is that development licensing procedures provide a public forum for considering the potential adverse environmental and social effects of a proposed aquaculture installation. These adversities must be assessed against the economic and

\footnotetext{
${ }^{20}$ Scottish Executive, Environment and Rural Development Department, Advice Note: Marine Fish Farming and the Environment, (Edinburgh Scottish Executive, 2003) (see http://scotland.gov.uk) para. 43 onwards.

21 SI 2002 No.193 and see Scottish Executive, Environment and Rural Development Department, What to do in the Event of an Escape of Fish from a Fish Farm, (Edinburgh Scottish Executive, 2002) (see http://scotland.gov.uk).

22 European Council Directive on the assessment of the effects of certain public and private projects on the environment (85/337/EEC) as now amended by Directive 97/11/EC.

${ }_{23}$ Scottish Executive, Environment and Rural Development Department, A Strategic Framework for Scottish Aquaculture (Draft), (Edinburgh Scottish Executive, 2002) (see http://scotland.gov.uk) para.3.71.
} 
developmental benefits that the project will be likely to secure. All parties with an interest in a aquaculture proposal have the opportunity to express their views and the determining body should strive to take these views into account in the final decision.

\section{Continuing Controls}

Development licensing and environmental assessment serve as invaluable mechanisms for evaluating projects before the commencement of an aquaculture operation. However, they have limitations in regulating the various subsequent activities which may take place at the site, and which need to be regulated by various kinds of continuing control. The kinds of continuing control that are needed will depend upon the nature of the installation and the location, but typically the following might be regulated by separate licensing requirements.

Water use licensing will be required, for example, where a land based operation is involved, to allow a sufficient quantity of water, of sufficient quality, to be drawn from available sources. Again, this kind of licensing is capable of being controversial in giving rise to conflicts with other water users who seek to use the same water for other purposes such as agriculture, industry or drinking water supply purposes. License applications will need to be openly considered so that scarce water resources are seen to be fairly allocated according to competing needs.

Wastewater discharge licensing is necessary when it is recognised that fish farms have the capacity to produce large amounts of waste products of various kinds which are capable of having seriously detrimental effects upon environmental quality and surrounding ecosystems. Equally, the discharge of poor quality effluent from a aquaculture installation may have a damaging impact upon other installations in the locality through transmission of contamination or disease, particularly where fish farms share a common watercourse or marine area. In relation to terrestrial fish ponds, with a discrete point of outflow, monitoring is relatively straightforward, but in relation to cage culture in open marine waters monitoring of effluent, and setting licence conditions, may be more problematic because it is not always clear what the 'discharge' refers to.

In Scotland, the difficulties of setting discharge consents for operations which involve treatment of fish for veterinary purposes, and inevitably involve natural dispersion of the chemicals involved, have been recognised by the environmental regulatory authority, the Scottish Environment Protection Agency. The approach adopted in Scotland is to adopt environmental quality standards for the receiving waters, which are set a level to ensure that concentrations of compounds are below that at which adverse ecological effects are detectable. Similarly, the problem of waste accumulating as bottom sediments below fish cages and consequent 'site souring' has necessitated a modified approach towards the setting of fish farm consents. Control of this problem requires a limitation of the biomass of fish cages to match the dispersive characteristics of the site, so that assimilative capacity is not 
exceeded. This process forms part of the assessment carried out by the Agency when determining discharge consent applications or reviewing existing consents. ${ }^{24}$

Chemical use licensing is another approach to the control of adverse impacts upon water quality. Various chemicals which are used in aquaculture, for veterinary, pest control or disinfection purposes, may need to be specifically controlled to avoid contamination of surrounding waters, by more direct mechanisms than wastewater discharge licensing. Hence, for control of particularly hazardous kinds of chemical restrictions may usefully be specifically imposed upon those chemicals. However, even where a risk is identified, a spectrum of legal responses are possible depending upon the gravity of the risk. In an extreme case, it is possible to impose total prohibition upon the possession of a particular chemical for use in aquaculture by creating appropriate criminal offence extending perhaps to the importing, distribution or sale of the chemical for aquaculture purposes. For less hazardous chemicals, it is possible to enact less severe control measures by making use subject to licensing requirements, veterinary prescription requirements or the adherence to requirements relating application of the substance, such as the certification of users or a code of practice governing use.

Fish movement licensing may be an appropriate response to a range of concerns that arise from aquaculture operations. In the first place, the collection of stock, particularly shellfish stock, from the wild may have a detrimental impact on natural stocks and ecosystems. In the second place, the escape of a farmed species into the wild may adversely impact upon local ecosystems through adverse genetic impacts upon native stocks or through invasive habitat competition. This may be seen as especially problematic where non-native species are involved and adverse ecological impacts are particularly difficult to assess. Finally, there is the significant threat of disease transmission, which is capable of having devastating economic consequences for the aquaculture industry as a whole. Equally important is the possibility of disease being transmitted from farmed to wild species.

For this range of reasons, control over fish movements is generally regarded as a legal essential for all but the most local, low intensity, aquaculture operations. Inevitably, controls must take the form of prohibitions or restrictions upon certain categories of fish movements, which will need to be made the subject of criminal offences. General prohibitions or restrictions upon movement will, however, need to be subject to a facility for allowing particular movements to be individually licensed where, after veterinary and ecological investigation, sufficient safeguards can be provided against the potential hazards that have been noted.

Fish movement licensing is invaluable as a means for securing prior assessment of risks and mitigation measures. Equally, it is of general benefit to the aquaculture industry in that it enables all significant movements to be recorded and information communicated to the relevant authority as a license condition. The monitoring of movements is important because it allows diseased stock, which has been the

\footnotetext{
${ }^{24}$ Scottish Executive, Environment and Rural Development Department, Advice Note: Marine Fish Farming and the Environment, (Edinburgh Scottish Executive, 2003) (see http://scotland.gov.uk) paras.39 and 40.
} 
subject of recent movements to be traced to other locations that may be infected. This enables swift action to be taken to contain the spread of the disease by imposing quarantine or slaughter requirements upon infected stocks. Officials will clearly need to be appropriately empowered to take whatever action is appropriate in a particular case, but without information from fish movement licensing there may be formidable difficulties in preventing or reducing the major hazard which disease represents to the aquaculture industry.

Fish movement licensing also has an important role in relation to the problem of escapes from fish farms, and in Scotland a working group from the Scottish Executive was set up to review the situation. It was concluded that there would always be circumstances, such as extreme weather conditions, which would result in escapes, but that improvements could be made in containment. In particular, the adequacy of site specific containment measures should be incorporated into the environmental assessment process and contingency plans for escapes should be included in development applications. Assessments of the suitability of containment measures should include a review of likely weather extremes and proposed net maintenance regimes in the light of operations to be conducted at a site. Pursuant to this, the industry has formulated a Code of Practice on the Containment of Farmed Fish. ${ }^{25}$ The Scottish Executive is also considering whether regulation is necessary to achieve minimum standards of cage design, equipment and maintenance, and to allow for prosecution for negligent acts or omissions resulting in escapes. ${ }^{26}$

Although the problem of escapes from fish farms has been recognised for some time, the extent of the problem has previously been rather speculative insofar as there has been no formal mechanism to record such incidents. In respect of Scotland, this position has recently been addressed by then enactment of regulations to require fish farmers to provide information about escapes. The Registration of Fish Farming and Shellfish Farming Businesses Amendment (Scotland) Order $2002^{27}$ amends existing requirements for the registration of fish farms to require the Scottish Ministers to be notified of the circumstances of any escape of fish, the number of fish involved and the number recovered. For the future, this allows the extent of the problem of fish escapes to be quantified, and for information to be gathered about the reasons for escapes. Nevertheless, the assessment of the impact of escapes remains a somewhat speculative exercise which may not be entirely dependent upon the numbers involved. The need, formally, to declare escapes is, at least, a beneficial first step in evaluating the ecological problem.

Licensing of genetically modified organisms may seem a little futuristic to some, but the technical capacity for modification of fish species, perhaps to enhance growth

\footnotetext{
${ }^{25}$ Scottish Executive, Environment and Rural Development Department, Advice Note: Marine Fish Farming and the Environment, (Edinburgh Scottish Executive, 2003) (see http://scotland.gov.uk) para.61 onwards.

26 Scottish Executive, Environment and Rural Development Department, A Strategic Framework for Scottish Aquaculture (Draft), (Edinburgh Scottish Executive, 2002) (see http://scotland.gov.uk) para.3.67.

27 SI 2002 No.193.
} 
rates or to improve disease resistance, is already on the horizon. Clearly, this is a matter of intense international controversy raising, profound environmental, ecological and developmental concerns, and the use of stock of this kind must be subject to the most careful scrutiny. Although fish movement licensing might be seen as an adequate mechanism for enabling thorough prior assessment of projects involving genetically modified fish, it may be thought more appropriate to deal with the issues under a separate system of controls which allows for more extensive and detailed scrutiny. Legislation, which fully reflects the breadth of environmental and public health concerns, would seem to be more appropriate than regulation within the sphere of aquaculture in isolation.

It may also be relevant to note that, for the future, the regulation of genetically modified organisms will need to be considered in the context of the Cartagena Protocol on Biosafety to the Convention on Biological Diversity (2000). The Biosafety Protocol aims at protecting biodiversity and consumers from any adverse impacts that could arise from transboundary movements of living modified organism and products derived therefrom. The Protocol has important implications for internationally traded products in requiring the exchange of information between exporting and the importing countries. Again, the gravity of the international issues which arise may be a sound reason for adopting a specialised national regime for genetically modified organisms, rather than attempting to deal with them in sectorspecific legislation.

Product quality licensing is also a regulatory essential insofar as fish products need to be regulated under public health legislation to avoid any potential hazard to the health of consumers. In times gone by, there may have been a tendency for food safety issues to be addressed at the point of consumer sale, increasingly the trend is to follow the food chain back into the distribution, harvesting and production processes. The vital importance of ensuring safety of aquaculture products throughout the production chain hardly needs to be reemphasised. A small number of contaminated products from a single producer are capable of having a devastating impact upon the industry as a whole.

To some extent, measures such as those directed at the misuse of chemicals in aquaculture may have important public health implications in preventing unacceptable residue levels in the final product. For the most part, however, public health is a technical and specialized area of regulation, which extends well beyond fish products in its compass. Food safety issues need to be addressed within a code of national law, which extends to food products of all kinds, so that common principles can be formulated, and consistent implementation and enforcement mechanisms applied. Not least important in this respect is the need for enforcement to be undertaken by an inspectorate with the specialised technical expertise needed to identify food safety issues in practice.

It needs also to be noted that food safety may give rise to specific concerns in relation to aquaculture insofar as products are intended for export. In particular, it may be necessary for national legislation to meet the requirements European Community Directive on Fish Products (91/493, as amended) 'laying down the health conditions for the production and placing on the market of fishery products' or 
the requirements of the United States Hazard Analysis and Critical Control Point (HACCP) system concerning procedures for the safe and sanitary processing and importing of fish and fishery products. ${ }^{28}$ Depending upon the intended export market, these measures will be of critical importance in determining the national standards for food safety in relation to aquaculture products. This issue is illustrative of the growing trend towards internationalisation of aquaculture legislation.

On licensing generally, the point is properly made that licensing systems are only as effective as the mechanisms which exist for inspection and checking that licence conditions are being adhered to, and the capacity of the enforcement authority to take action where they are not. The issue of enforcement is returned to later in the discussion.

\section{Consolidated Regulation and Integrated Strategy}

From the perspective of a prospective fish farmer, regulation can seem an insuperable obstacle, and the scan of possible approaches to licensing that has been provided should leave no doubt as to the potential complexities involved. The range of potential impacts of aquaculture, leads into a range of distinct fields of regulation each with its own peculiarities and intricacy. An important issue to be addressed is the extent to which laws governing aquaculture are capable of being consolidated into a single statute, or at least a small number of legal provisions. In an ideal world, it might be thought possible to bring all aquaculture operations within a comprehensive regime which allowed for a single licence or authorisation governing all kinds of impacts. Such an approach would avoid the need for multiple applications to different licensing authorities for slightly different purposes, with corresponding multiplication of the bureaucracy involved.

Although consolidated or codified legislative provision for aquaculture seems a generally desirable policy goal, the reality is that it would be tremendously difficult to achieve. Environmental quality issues, public health concerns and activities such as the cultivation of genetically modified species, are not matters that are unique to aquaculture and need to be addressed consistently across different sectors of activity. For this reason, an 'all-purpose aquaculture licence' would be formidably difficult to achieve, for anything but the most low intensity small scale project. Nonetheless, there should be scope for the administrative co-ordination of activities of regulatory authorities. This might be achieved by 'one stop' licensing, whereby the key authority to which an application is submitted has the responsibility of liaising with other regulatory bodies that are involved to secure that the full range of authorisations are secured. From a fish farmer's point of view, this approach has obvious attractions in minimising administrative complexity and requiring regulators to sort of different kinds of regulatory requirements between themselves.

${ }^{28}$ Generally see E. Ruckes, 'Aquaculture Products Quality and Marketing' in R. Leroy Creswell and R. Flos, Eds., Perspectives on Responsible Aquaculture for the New Millennium (Louisiana and Oostende: World Aquaculture Society and European Aquaculture Society, 2002) p.63. 
Whether or not regulatory or administrative consolidation can be achieved, it is essential that governments provide an overall strategy for aquaculture, which plots out the development of the industry and the mechanisms by which development is to be accomplished. Clearly, national strategies will be largely determined by national circumstances and perceptions of priority. However, an illustrative example of a national strategy is to be seen in the Scottish Executive's recently produced a draft Strategic Framework for Scottish Aquaculture. ${ }^{29}$ This is a wide ranging document which seeks to formulate the respective roles of government, the industry and other stakeholders in aquaculture development across a wide range of issues. The shared vision is that:

"Scotland will have a sustainable, diverse competitive and economically viable aquaculture industry, of which its people can be justifiably proud. It will deliver high quality, healthy food to consumers at home and abroad, and social and economic benefits to communities, particularly in rural and remote areas. It will operate responsibly, working within the carrying capacity of the environment, both locally and nationally and throughout its supply chain."

Revealingly, the draft Strategy reports the contention of the industry that costs are higher than those of competitors in other countries because of inefficiencies of scale resulting from regulatory constraints. ${ }^{30}$ The Scottish Executive is considering undertaking comparative research to assess the extent to which regulation actually does place Scottish aquaculture at a competitive disadvantage.

It is also notable, in the United Kingdom context, that strategy is a matter which will increasingly need to be addressed at a regional international level, and a strategic position on aquaculture is being developed by the European Community. The Commission has recently published a communication document, $A$ Strategy for the Sustainable Development of European Aquaculture ${ }^{31}$ which builds upon previous Community initiatives that have provided support for the sector. The document affirms the potential for aquaculture development but also notes problems in relation to health protection requirements, environmental impact and market instability. The fundamental issue is perceived to be the maintenance of competitiveness, productivity and durability for the sector, recognising that further development must take a more integrated approach to farming technologies, socio-economics, natural resource use and governance. Specific legislation relating to aquaculture does not exist at Community level, though many measures at Community level have significant implications for aquaculture. However, reliance upon national legislation has been alleged to lead to competition distortions, suggesting that this is an area on which the Community might seek to legislate comprehensively at some stage in the future.

\footnotetext{
${ }^{29}$ Scottish Executive, Environment and Rural Development Department, A Strategic Framework for Scottish Aquaculture (Draft), (Edinburgh Scottish Executive, 2002) (see http://scotland.gov.uk). It is understood that the final version of the strategy is imminent.

${ }^{30}$ Scottish Executive, Environment and Rural Development Department, A Strategic Framework for Scottish Aquaculture (Draft), (Edinburgh Scottish Executive, 2002) (see http://scotland.gov.uk) para.3.4.

${ }^{31}$ European Commission, A Strategy for the Sustainable Development of European Aquaculture COM (2002) 511 final (Brussels: European Commission, 2002).
} 


\section{Implementation and Enforcement}

Despite the general coincidence between sustainability and aquaculture that has been previously referred to, there have been regrettable examples of bad practice where aquaculture has damaged itself through pollution, inadequate disease control and practices which have harmed the food safety reputation of aquaculture products generally. Regulation is needed to outlaw the activities of the small number of participants who might wish to secure short-term gains, at the expense of the reputation of the industry as a whole, both to protect the environment which it depends and to secure the quality of the products which it produces.

In respect of those activities that are sufficiently serious to be subject to prohibition, the most vital consideration is that of enforceability. There is little point in enacting laws that cannot be enforced in practice, or enacting laws without careful prior consideration of the allocation of responsibilities for enforcement. On this, the relatively specialised nature of aquaculture legislation should be recognised. Aquaculture law is different from laws governing agriculture, fishery activities or laws governing water quality generally. The counterpart of this is that enacting legislation implies a commitment to the establishment and support of specialised inspection and enforcement agencies which are necessary to ensure proper implementation.

The problem, all too frequently, is that legislators are inclined to make laws without sufficient thought to the resources and costs which are necessary for their implementation and enforcement. Arguably, the thinking process should be put in reverse since there is little point in enacting regulation if, in practice, the means to ensure that it will be properly applied are lacking. Moreover, the cost of implementing and enforcing legislation should be put into perspective by comparison with the potential costs of failing to enforce legal requirements effectively, especially where this has the result of inflicting massive economic damage upon the industry itself through the failure to control disease or to achieve product control requirements because of contamination.

The highly specialised nature of aquaculture legislation should be emphasised. Controls upon fish movements, for example, are generally thought necessary to prevent the transmission of disease between fish farms and to prevent the introduction of non-native species which are likely to give rise to ecologically damaging habitat competition or loss of genetic integrity of native stocks. However, the practicalities involved in securing actual control of individual fish movements are not to be underestimated. Unavoidably, a fairly complex administrative structure is required to secure the registration of origins and destination of fish stocks in relation to all relevant fish movements. The enactment of regulation presupposes the existence, or provision, of fairly intricate administrative structures to ensure that the legal requirements operate effectively. Without such controls, legislation has little prospect of fulfilling its objectives.

Implementation of regulation needs to be considered before laws are enacted, rather than afterwards. The process of prior regulatory appraisal has rapidly gained ground in many jurisdictions, involving an explicit attempt by legislators to assess the continuing administrative and enforcement costs to which regulation will give 
rise and to confirm that these costs will actually be met. It is suggested that the prior regulatory appraisal process has much to commend it in the context of aquaculture legislation given the particularly specialised nature of administration and enforcement requirements that are entailed.

Beyond regulatory impact appraisal, reflection is needed upon the status and powers of the agency or authority which is responsible for implementation. The importance of providing an appropriate level of resourcing of a regulatory agency has been noted, but another important consideration is the degree of independence with which the agency operates. Clearly conflicts of interest between regulators and regulated bodies must be avoided, but also it might be seen as desirable to secure some degree of independence between regulatory authorities and government. The role of government is to formulate policies for the development and promotion of the aquaculture sector and to provide the resources necessary to realise those policy objectives. The role of a regulatory authority is to enforce laws effectively and impartially. For this reason, some degree of separation of powers between a government and a aquaculture regulatory authority might be seen as a desirable feature.

In the United Kingdom context there are various mechanisms which secure a degree of insulation between government and practical regulation of fish farms. It is fair to note that some regulatory functions are exercised through government departments. In particular, the responsibilities for securing that works in tidal waters do not constitute a hazard to navigation ${ }^{32}$ are allocated to transport ministries. ${ }^{33}$ Similarly responsibilities for measures concerning fish health ${ }^{34}$ are allocated at central government level. ${ }^{35}$ These provisions concern registration of fish farms and provide for actions to be taken in relation to notified diseases. ${ }^{36}$ However, perhaps the most important regulatory powers in relation to fish farms are allocated to the Scottish Environment Protection Agency. The Agency has the key power to determine applications for discharge consents for fish farms and may impose conditions to minimise adverse environmental impacts. Such consents are determined against a general duty to promote the cleanliness of waters and to have regard to the need to conserve flora and fauna. The significant point here is that the Agency has its powers and duties determined by statute, but actually has a fair degree of independence from government in determining any particular discharge consent application.

Similar observations could be made about the role of the nature conservation authority, Scottish Natural Heritage, which, again, has a reasonable degree of independence from government in making representations about the appropriateness of fish farm development applications in respect of nature conservation and landscape impacts. Also, the transfer of land use planning powers

32 Under the Coast Protection Act 1949.

33 In Scotland, to the Scottish Executive Development Department Transport Division.

34 Under the Diseases of Fish Act 1937 and 1983, and European Community fish health legislation.

35 In Scotland, to the Scottish Executive Environment and Rural Affairs Department.

36 Scottish Executive, Environment and Rural Development Department, Advice Note: Marine Fish Farming and the Environment, (Edinburgh Scottish Executive, 2003) (see http://scotland.gov.uk) para.18 onwards. 
to local authorities, that is imminent in Scotland in relation to fish farm applications, will give local authorities quite a degree of autonomy from government in making individual determinations. The transfer of these powers to bodies which operate with some degree of independence from government, has the consequence that any pro-development tendencies of government are capable of being moderated by bodies with specialised sectoral remits. ${ }^{37}$

\section{Concluding Observations}

Successful aquaculture regulation involves reconciling the interests of aquaculture participants, the communities in which they are based, the consumers of their products and those who advocate vigilance over the environment in which aquaculture takes place. These interests are not coincident, but should be capable of reconciliation. The consensus is that aquaculture is an activity with such tremendous potential for human benefit, and that mechanisms have to be found to facilitate its development in a way that respects the competing concerns that have been referred to.

${ }^{37}$ Scottish Executive, Environment and Rural Development Department, Advice Note: Marine Fish Farming and the Environment, (Edinburgh Scottish Executive, 2003) (see http://scotland.gov.uk) para. 25 onwards. 